\title{
Vibrationally resolved rate coefficients and branching fractions in the dissociative recombination of $\mathrm{O}_{2}^{+}$
}

\author{
Annemieke Petrignani ${ }^{\mathrm{a})}$ \\ FOM Institute for Atomic and Molecular Physics, Kruislaan 407, 1098 SJ, Amsterdam, The Netherlands \\ Wim J. van der Zande \\ Molecule and Laser Physics, IMM, Radboud University Nijmegen, Toernooiveld 1, 6525 ED Nijmegen, \\ The Netherlands \\ Philip C. Cosby \\ Molecular Physics Laboratory, SRI International, Menlo Park, California 94025 \\ Fredrik Hellberg, Richard D. Thomas, and Mats Larsson \\ Molecular Physics, Stockholm University, Albanova University Centre, SE 10691 Stockholm, Sweden
}

(Received 9 July 2004; accepted 7 October 2004; published online 13 December 2004)

\begin{abstract}
We have studied the dissociative recombination of the first three vibrational levels of $\mathrm{O}_{2}^{+}$in its electronic ground $X^{2} \Pi_{g}$ state. Absolute rate coefficients, cross sections, quantum yields and branching fractions have been determined in a merged-beam experiment in the heavy-ion storage ring, CRYRING, employing fragment imaging for the reaction dynamics. We present the absolute total rate coefficients as function of collision energies up to $0.4 \mathrm{eV}$ for five different vibrational populations of the ion beam, as well as the partial (vibrationally resolved) rate coefficients and the branching fractions near $0 \mathrm{eV}$ collision energy for the vibrational levels $v=0,1$, and 2 . The vibrational populations used were produced in a modified electron impact ion source, which has been calibrated using $\mathrm{Cs}-\mathrm{O}_{2}^{+}$dissociative charge transfer reactions. The measurements indicate that at low collision energies, the total rate coefficient is weakly dependent on the vibrational excitation. The calculated thermal rate coefficient at $300 \mathrm{~K}$ decreases upon vibrational excitation. The partial rate coefficients as well as the partial branching fractions are found to be strongly dependent on the vibrational level. The partial rate coefficient is the fastest for $v=0$ and goes down by a factor of two or more for $v=1$ and 2 . The $\mathrm{O}\left({ }^{1} S\right)$ quantum yield, linked to the green airglow, increases strongly upon increasing vibrational level. The effects of the dissociative recombination reactions and super elastic collisions on the vibrational populations are discussed. (C) 2005 American Institute of Physics. [DOI: 10.1063/1.1825991]
\end{abstract}

\section{INTRODUCTION}

Dissociative recombination (DR) is an important reaction for the Earth's atmosphere. It is a sink for low-energy electrons and a source for airglows and highly reactive and kinetic product atoms. The DR of $\mathrm{O}_{2}^{+}$is considered to be the only source for the green airglow in the nighttime F-region of our ionosphere. The $\mathrm{O}\left({ }^{1} S\right)$ atoms formed in the DR reaction produce the green airglow at $557.7 \mathrm{~nm}$ when they relax radiatively to the $\mathrm{O}\left({ }^{1} D\right)$ state, while the $\mathrm{O}\left({ }^{1} D\right)$ atoms give rise to the red airglow at $630.0 \mathrm{~nm}$ when relaxing to the ground state. The exothermicity of the DR reaction is significant and influences heating, gravitational escape and the terrestrial geo-corona. ${ }^{1,2}$ Understanding the DR reaction will enable us to make predictions on airglows and their related variables; the product atom quantum yields and the DR rates both depend on the rovibrational state of the molecular ions as well as on the kinetic temperature of both the electrons and the ions. Airglows could become a useful remote sensing tool when these dependencies and the mechanisms of DR are well known and understood.

${ }^{\text {a)} E l e c t r o n i c ~ m a i l: ~ a . p e t r i g n a n i @ a m o l f . n l ~}$
There are numerous previous atmospheric, laboratory and theoretical papers on both the DR rate coefficients and branching of $\mathrm{O}_{2}^{+}$. The in situ research varies from groundbased and air-borne operations to satellite based instruments and space probes. The laboratory research uses ion traps, stationary and flowing afterglows and merged-beams techniques. The absence of accurate knowledge on the internal vibrational energy of the molecular oxygen ions has hindered the interpretation of both laboratory research as well as in situ data.

The thermal rate coefficient, $\alpha\left(\mathrm{O}_{2}^{+}\right)$, is well established and reproduced in many experiments. The reported laboratory values ${ }^{3-13}$ range from $1.7 \cdot 10^{-7}$ to $2.4 \cdot 10^{-7} \mathrm{~cm}^{3} \mathrm{~s}^{-1}$ at $300 \mathrm{~K}$. The commonly used value for ground-state oxygen ions at $300 \mathrm{~K}$ is $1.95 \cdot 10^{-7} \mathrm{~cm}^{3} \mathrm{~s}^{-1}$. In general, the temperature dependence of the thermal rate coefficient is reported to follow $\alpha\left(\mathrm{O}_{2}^{+}\right) \sim T^{-0.7}$ with $T$ pertaining mainly to the electron temperature. In merged-beam experiments it is the rate coefficient, $k$, in terms of the electron collision energy which is commonly reported and its dependence is found ${ }^{13-16}$ to be $k\left(\mathrm{O}_{2}^{+}\right) \sim E_{c}^{-0.5}$. The agreement on the thermal rate coefficient is somewhat surprising in view of the fact that many of these experiments had unknown and certainly often different 
vibrational populations of the parent ions. The branching fractions and quantum yields of the product atoms for different vibrational levels is not well established and little is known on any vibrational dependence. In the case of the $\mathrm{O}\left({ }^{1} S\right)$ quantum yield, experimental values have been found ranging from almost $0.7 \%$ to $10 \% .^{9,17-21}$ Again, many of these experiments had different, and often unknown, vibrational populations of the parent ions. Some consensus has been reached for the branching fractions of the vibrational ground state of $\mathrm{O}_{2}^{+}\left(X^{2} \Pi_{g}\right)$ near $0 \mathrm{eV}$ collision energy. These fractions have been determined using ion storage rings, including the results reported here. At the ASTRID ion storage ring, a beam of an unknown vibrational composition of the mixed isotope ${ }^{16} \mathrm{O}^{18} \mathrm{O}^{+}$was used in the studies. ${ }^{20}$ In a more recent experiment at the CRYRING ion storage ring, an $\mathrm{O}_{2}^{+}$ beam was used that consisted of $v=0$ ions only. ${ }^{21}$ The corresponding $\mathrm{O}\left({ }^{1} S\right)$ quantum yields are in good agreement with the theoretical estimates. In a recent theoretical paper ${ }^{22}$ on the DR of $\mathrm{O}_{2}^{+}$, Guberman predicts an $\mathrm{O}\left({ }^{1} S\right)$ quantum yield of between $1.6 \%$ and $2.9 \%$ from the reaction of $v=0$ ground-state oxygen ions after introducing spin-orbit coupling into the model. The inclusion of spin-orbit coupling was a first for the modeling of a DR reaction. These predicted quantum yields are relatively large; in an earlier paper Guberman ${ }^{23}$ predicted a negligible $\mathrm{O}\left({ }^{1} S\right)$ cross section for $\mathrm{O}_{2}^{+} v=0$ ions and small $\left.\mathrm{O}^{1} S\right)$ cross sections from $v=1$ and 2 ions. Additional experimental data exists on the collisionenergy dependence of the branching fractions of the $\mathrm{O}_{2}^{+}\left(X^{2} \Pi_{g}, v=0\right)$ ions. ${ }^{13,24}$ Although theory is making considerable progress, to the best of our knowledge, no complete branching results have been reported for the DR of $\mathrm{O}_{2}^{+}(v$ $\geqslant 0$ ).

In the case of atmospheric observations, many data are reported in which variations in the green airglow produced by de-excitation of $\mathrm{O}\left({ }^{1} S\right)$ are linked to changes in the vibrational excitation of the parent $\mathrm{O}_{2}^{+}$ions. The change of $2 \%-$ $23 \%$ in the $\mathrm{O}\left({ }^{1} S\right)$ quantum yield predicted from in situ measurement ${ }^{25-33}$ is even larger than that suggested from the laboratory experiments. From these in situ observations, it is believed that the $\mathrm{O}\left({ }^{1} S\right)$ yield must be strongly dependent on the vibrational states of the parent $\mathrm{O}_{2}^{+}$ion ${ }^{31-34}$ with the vibrational excitation of the $\mathrm{O}_{2}^{+}$ions believed to increase with altitude and the largest $\mathrm{O}\left({ }^{1} S\right)$ yields to come from the highest vibrational states. Studies on the production and quenching of $\mathrm{O}_{2}^{+}(v)$ in the ionosphere, however, often report the vibrational deactivation at nighttime is fast, giving a source of confusion concerning the role of DR in the production of $\mathrm{O}\left({ }^{1} S\right)$ in the F-region of our ionosphere. ${ }^{9,35-37}$ The role of DR in planetary atmospheres is also ill understood. For a long time it was believed that the green airglow could be a diagnostic tool for the detection of $\mathrm{O}_{2}$ bearing atmospheres. However, in 2001, Slanger et al. reported the discovery of the oxygen green line in the Venus night glow, ${ }^{38}$ while Venus does not have an Earth-like atmosphere. The production of $\mathrm{O}\left({ }^{1} S\right)$ responsible for the Venusian green-line emission is attributed to another reaction at lower altitudes. No vibrational dependent data on DR was available to include its possible influence.

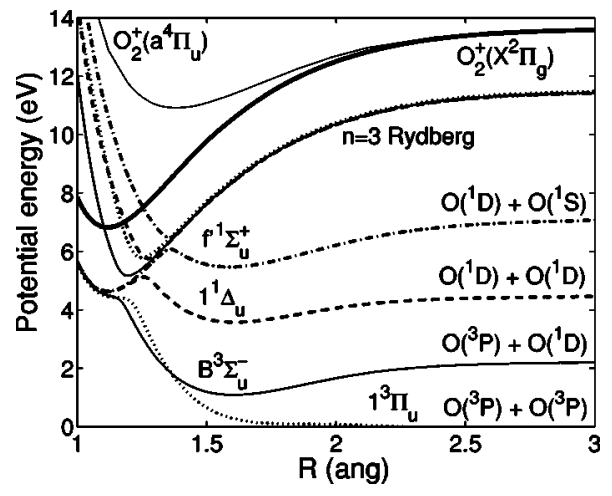

FIG. 1. Schematic of the potential curves relevant for the DR process with $\mathrm{O}_{2}^{+}$. The electronic ground state $X^{2} \Pi_{g}$ and the first ionic metastable state $a^{4} \Pi_{u}$ are given. A number of neutral capture states is given in an adiabatic representation showing the consequences of the interaction with the $n=3$ Rydberg states. The dissociation limits relevant for this work are given on the right hand side.

\section{A. The DR reaction with $\mathrm{O}_{2}^{+}$}

At $0 \mathrm{eV}$ collision energy there are five dissociation limits energetically open [see Eqs. (1a)-(1e)] for the DR of ground-state $\mathrm{O}_{2}^{+}\left(X^{2} \Pi_{g}, v=0\right)$. The next dissociation limit [see Eq. (1f)] requires $1.41 \mathrm{eV}$ of energy, either from the incoming electron in the form of collision energy $E_{c}$ or from internal excitation of the $\mathrm{O}_{2}^{+}$. In the latter case this internal energy, $E_{v}$, is not reached until $\mathrm{O}_{2}^{+}\left(X^{2} \Pi_{g}, v=8\right)$.

$$
\begin{aligned}
\mathrm{O}_{2}^{+} & \left(X^{2} \Pi_{g}, v\right)+e^{-}\left(E_{c}\right) \\
& \rightarrow \mathrm{O}\left({ }^{3} P\right)+\mathrm{O}\left({ }^{3} P\right)+\left(6.95+E_{v}+E_{c}\right) \mathrm{eV}, \\
& \rightarrow \mathrm{O}\left({ }^{3} P\right)+\mathrm{O}\left({ }^{1} D\right)+\left(4.99+E_{v}+E_{c}\right) \mathrm{eV}, \\
& \rightarrow \mathrm{O}\left({ }^{1} D\right)+\mathrm{O}\left({ }^{1} D\right)+\left(3.02+E_{v}+E_{c}\right) \mathrm{eV}, \\
& \rightarrow \mathrm{O}\left({ }^{3} P\right)+\mathrm{O}\left({ }^{1} S\right)+\left(2.77+E_{v}+E_{c}\right) \mathrm{eV}, \\
& \rightarrow \mathrm{O}\left({ }^{1} D\right)+\mathrm{O}\left({ }^{1} S\right)+\left(0.80+E_{v}+E_{c}\right) \mathrm{eV}, \\
& \rightarrow \mathrm{O}\left({ }^{1} S\right)+\mathrm{O}\left({ }^{1} S\right)+\left(-1.41+E_{v}+E_{c}\right) \mathrm{eV} .
\end{aligned}
$$

Figure 1 shows a number of relevant potential curves for both neutral and ionic molecular oxygen. The dissociation limits are shown on the right hand side. The potential diagram reveals some of the possible complications in assessing the outcome of the DR reaction in a simple way. First, the avoided crossings between the different repulsive states and the low lying Rydberg states indicate large electronic couplings between the associated diabatic states, and those need to be taken into account when trying to predict the branching fractions and rate coefficients. Second, the production of the $\mathrm{O}\left({ }^{1} S\right)$ products is complex. The $\mathrm{O}\left({ }^{1} D\right)+\mathrm{O}\left({ }^{1} S\right)$ dissociation limit [see Eq. (1e)] is assumed to be the only channel leading to $\mathrm{O}\left({ }^{1} S\right)$ products, via the repulsive ${ }^{1} \Sigma_{u}^{+}$state. ${ }^{23,39}$ For ground-state $\mathrm{O}_{2}^{+}$, this channel is neither accessible via the direct nor the indirect mechanisms, thus reducing the $\mathrm{O}\left({ }^{1} S\right)$ yield. ${ }^{40}$ The route to this channel is via formation of Rydberg states in the ${ }^{3} \Sigma_{u}^{-}$manifold followed by a radiationless transition involving spin-orbit coupling. ${ }^{22}$ For higher vibrational levels the direct route to the ${ }^{1} \Sigma_{u}^{+}$state is open. Strong indications exist that the $\mathrm{O}\left({ }^{3} P\right)+\mathrm{O}\left({ }^{1} S\right)$ dissociation limit [see 
(a)



(b)

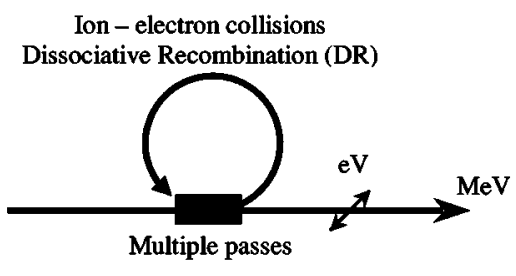

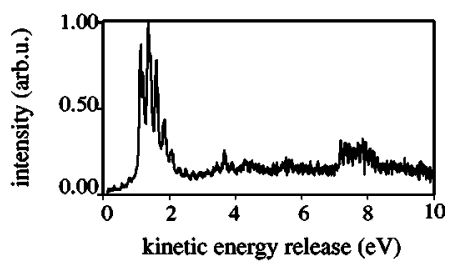



FIG. 2. Schematic diagram of (a) the dissociative charge transfer (DCT) experiment used to characterize the five vibrational populations of $\mathrm{O}_{2}^{+}$used in the DR experiment shown in (b).
Eq. (1d)] does not play a role in the DR process. These indications are found both in experiments with vibrational ground-state $\mathrm{O}_{2}^{+}$ions and low energy electrons ${ }^{13,24}$ as in an experiment ${ }^{20}$ with the isotopomer ${ }^{16} \mathrm{O}^{18} \mathrm{O}^{+}$. Additionally Helm et al. ${ }^{19}$ did not observe any dissociation towards the $\mathrm{O}\left({ }^{3} P\right)+\mathrm{O}\left({ }^{1} S\right)$ channel for all vibrations $v=0-6$ in a process of field induced predissociation of high lying Rydberg states. This process has similarities with the DR process. Calculations indicate that the lowest lying repulsive state that dissociates to the $\mathrm{O}\left({ }^{3} P\right)+\mathrm{O}\left({ }^{1} S\right)$ limit crosses the ionic ground state near the $v=9$ level. $^{23,41}$

The motivation of the present research is to provide new laboratory answers to the question on the vibrational dependence of the DR reaction. In this paper we report on the effect of vibrational excitation on the DR reaction of $\mathrm{O}_{2}^{+}$. We first discuss the control over the vibrational populations of the parent oxygen ions that is necessary to unravel these dependencies. The total rate coefficients for collision energies up to $0.4 \mathrm{eV}$ using ion beams with five different vibrational populations are presented and discussed. Finally we report on the product distributions using these same vibrational populations at $0 \mathrm{eV}$ collision energy, deriving partial (vibrationally resolved) rate coefficients, quantum yields and branching fractions for $\mathrm{O}_{2}^{+}\left(X^{2} \Pi_{g}, v=0-2\right)$.

\section{EXPERIMENT}

The experimental work was conducted at SRI International, California and at the heavy ion storage ring, CRYRING, at the Manne Siegbahn Laboratory, Stockholm. The ion source used in all experiments was constructed at the FOM Institute for Atomic and Molecular Physics (FOMAMOLF), Amsterdam. At SRI several different vibrational populations produced by the ion source were characterized using dissociative charge transfer (DCT) reactions between cesium and oxygen (see Sec. II A). These populations were then reproduced in the CRYRING (see Sec. IIB) and for each ion-beam population the DR rate as function of collision energy together with the product distributions at $0 \mathrm{eV}$ collision energy were investigated.

The DCT and the DR experiments both employ a fast ion beam combined with an imaging technique to determine the kinetic energy released in the respective reactions. Figure 2(a) sketches the DCT apparatus used in the characterization of the vibrational populations, and Fig. 2(b) shows the experimental arrangement employed in the DR study. The DCT studies are a single-pass experiment, in which the ion-beam energy is a few $\mathrm{keV}$ and the interaction region is small (a few $\mathrm{mm}$ ) and extremely well defined. The DR experiments are conducted at $\mathrm{MeV}$ energies and, since the ions circulate in the storage ring, they pass through the $85 \mathrm{~cm}$ interaction region multiple times. The imaging technique in the DCT experiment involves the coincident detection of both oxygen fragments, recording their position and difference in arrival times (so-called 3D-detection). The vibrational population in the parent ions can be observed directly. However, in the DR experiment the high beam energy means that extracting the arrival-time difference (ns) is nontrivial ${ }^{13,42}$ and so only the positions of the fragments were recorded. The acquired data is therefore a $2 \mathrm{D}$ projection of the dissociation which, together with the long interaction region, limits the experimental energy resolution. The vibrational structure is embedded underneath the electronic structure. Another important difference between the DCT and DR studies is their time structure. The ions in the DCT experiment are accelerated on their way to the collision cell and the charge transfer reaction takes place some tens of $\mu$ s after their formation. In the DR storage ring experiment, the $\mathrm{O}_{2}^{+}$ions have to be accelerated to the required $\mathrm{MeV}$ energy before the experiment begins and this typically takes about $1.1 \mathrm{~s}$.

\section{A. Production and characterization of vibrational populations}

In order to study vibrational dependencies of the DR reaction, control over the vibrational population of the parent ions is necessary. For this purpose a Nier type electron impact ion source has been developed. A similar source has been used in previous research on the DCT reactions of molecular ions with cesium. ${ }^{19,43,44}$ Figure 3 shows a schematic of the ion source. Oxygen enters through the gas inlet, where the flow rate is monitored to accurately control the pressure inside the source. The filament producing the electrons is external to the source to reduce the heat load on the gas and the ion source is further cooled by water. A magnetic guiding field is used to direct the electrons emitted by the filament to the electron trap. The voltage difference between the filament 


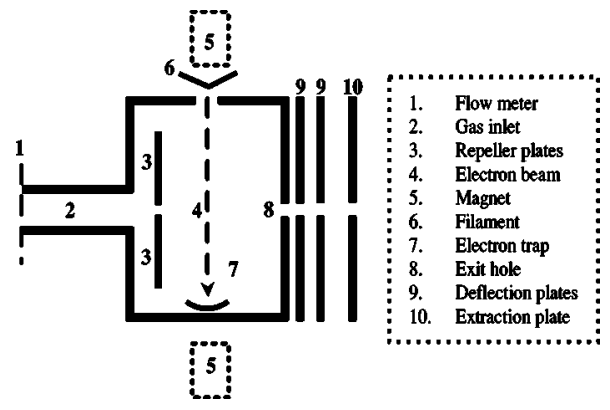

FIG. 3. Schematic diagram of the electron impact ion source designed to control the creation of different vibrational populations of $\mathrm{O}_{2}^{+}$ions.

and the trap determines the electron impact energy. Two repeller plates located inside the source are used to steer the ions towards the exit aperture and thus effect their residence time inside the source. Finally, the deflection and extraction plates are used to guide and accelerate the exiting ion beam.

The vibrational population of the ions leaving the ion source is controlled by manipulating the conditions inside the source. By varying the electron impact energy, the pressure, and the voltage on the repeller plates, we can effect the formation and interactions of the ions. Raising the electron impact energy increases the excitation of the ions, whereas increasing the pressure or decreasing the repeller voltages increases the collisional quenching of the ions. Each combination of these variables creates a specific vibrational population. Consequently, a certain vibrational population can be reproduced by switching to its corresponding ion-source setting.

In order to characterize several vibrational populations, we looked at the DCT reaction between the oxygen ions and cesium. ${ }^{19,43,45,46}$ Five vibrational populations associated with five distinct ion-source settings were chosen such that the vibrational excitation gradually increased. For each ionsource setting, the $\mathrm{O}_{2}^{+}$ions were extracted, magnetically mass selected and accelerated to $5 \mathrm{keV}$ before entering a collision cell containing Cs vapor [see Fig. 2(a)]. The DCT reactions taking place in the collision cell are

$$
\begin{aligned}
\mathrm{O}_{2}^{+}\left(X^{2} \Pi_{g}, v\right) & \stackrel{\text { Cs }}{\rightarrow} \mathrm{O}_{2}^{*}\left(3 s \sigma^{1,3} \Pi_{g}, v^{\prime}=v\right) \\
& \rightarrow \mathrm{O}\left({ }^{3} P\right)+\mathrm{O}\left({ }^{1} D\right)+\left(\sim 1+E_{v}\right) \quad e V, \\
& \rightarrow \mathrm{O}\left({ }^{3} P\right)+\mathrm{O}\left({ }^{1} D\right)+\left(\sim 3+E_{v}\right) \quad e V, \\
& \rightarrow \mathrm{O}\left({ }^{1} D\right)+\mathrm{O}\left({ }^{1} D\right)+\left(\sim-1+E_{v}\right) \quad e V
\end{aligned}
$$

Of relevance to the present investigation is the conservation of the vibrational quantum number in going from the parent ion to the $3 s$ Rydberg states. This conservation gives rise to spectra with kinetic energy releases (KERs) which mirror directly the vibrational structure of the $\mathrm{O}_{2}^{+}$parent ions. For $v=0$ ions, the KER of Eqs. (2a) and (2b) is around 1 and 3 $\mathrm{eV}$, respectively. Equation (2c) is only energetically possible for $v>3$. Whenever metastable $\mathrm{O}_{2}^{+}\left(a^{4} \Pi_{u}\right)$ ions are produced, reactions with this state will also be observed, since there will be insufficient time for the metastable ion to decay before it reaches the collision cell. The KER then observed is around $7 \mathrm{eV}$ and higher:

$$
\begin{aligned}
\mathrm{O}_{2}^{+}\left(a^{4} \Pi_{u}, v\right) & \stackrel{\text { Cs }}{\rightarrow} \mathrm{O}_{2}^{*}\left(R y d, v^{\prime}=v\right) \\
& \left.\rightarrow \mathrm{O}\left({ }^{3} P\right)+\mathrm{O}^{3} P\right)+\left(\sim 7+E_{v}\right) \quad \mathrm{eV} .
\end{aligned}
$$

The resolution of the DCT experiment is more than sufficient to easily distinguish between the vibrational levels. By integration of the data in the DCT fragmentation spectra, the vibrational population of the $\mathrm{O}_{2}^{+}$ions created in the ion source can be determined directly. Explicit details on the DCT reaction between oxygen and cesium and the data analysis can be found elsewhere. ${ }^{46,47}$

\section{B. Dissociative recombination rates and dynamics}

The DR experiment is a merged-beam experiment carried out at the CRYRING heavy-ion storage ring. Details of CRYRING and the imaging technique employed can be found elsewhere in the literature. ${ }^{13,48,49}$ Here, only the main and most relevant features will be outlined. The ion source described earlier was integrated into the injectionline apparatus. The five vibrational populations characterized in the DCT experiment were reproduced using their corresponding ion-source settings. The $\mathrm{O}_{2}^{+}$ions extracted from the source were magnetically mass selected and injected into the storage ring at the platform energy of $40 \mathrm{keV}$. The injection denotes the start of the ring cycle. The ions were accelerated in $1.1 \mathrm{~s}$ to the full beam energy of $3.05 \mathrm{MeV}$ after which they were allowed to coast in the ring. During each revolution, the ions are merged with an electron beam in one of the straight sections in the ring, the so-called electron cooler. The electron cooler has a length of $0.85 \mathrm{~m}$ and the total circumference of the storage ring is $51.6 \mathrm{~m}$. The ion beam passes through the electron-cooler many times during its storage time. Each time the ions pass the electron cooler, interaction with the cold electron beam results in a transversal cooling of the ions. ${ }^{48}$ The interaction region acts as the site for the DR reactions. Other possible interactions in the merged beam section are elastic and super-elastic collisions (SECs) between the ions and the electrons. ${ }^{50,51}$ Dissociative charge transfer reactions ${ }^{13}$ between the ions and the rest gas occur throughout the ring. These charge transfer reactions produce two neutral product atoms, as in DR, and therefore constitute a background signal which must be accounted for. This background signal is determined with no or negligible DR signal present, i.e., without electrons or at very high collision energies, respectively. SEC reactions may cool the vibrational population in the ring and the impact of these reactions is an uncertain factor in the experiments, since they could create a time dependence of the vibrational population of the $\mathrm{O}_{2}^{+}$ ions. We have assessed the importance of the SEC process by studying the DR reaction at different storage times (see Sec. III B).

\section{Dissociative recombination rates: Total rate coefficients}

In the first part of the DR experiment the reaction rate for each of the ion-source settings was measured as function of collision energy up to $0.4 \mathrm{eV}$. A surface barrier detector situated in the zero-degree arm following the electron cooler has been used to detect the neutral atoms produced in the DR 
reaction. ${ }^{13}$ Measurements were taken during the full storage time. The collision energy was ramped between 0 and $0.4 \mathrm{eV}$ both by accelerating and decelerating the electron beam with respect to the ion beam, ensuring that a collision energy of 0 $\mathrm{eV}$ is achieved. The ramp started after $2.25 \mathrm{~s}$ storage time and lasted for $1.5 \mathrm{~s}$. The count rate due to background reactions was measured independently for each population by turning the collision energy briefly up to $5 \mathrm{eV}$, where the DR cross section is negligible. After background subtraction, the DR signal was further corrected to account for the gradual decrease of the ion beam intensity in the ring, providing us with the DR count rate as function of time. The time axis was transformed to collision energy, taking into consideration the necessary corrections, ${ }^{52}$ and the DR count rate was further transformed to the total rate coefficient $k=\langle\sigma v\rangle$, also after applying the necessary corrections. ${ }^{53,54}$

\section{Dissociative recombination dynamics: Partial rate coefficients and branching fractions}

In the studies on the DR dynamics we restricted the collision energy to a nominal $0 \mathrm{eV}$. That means the collision energy was set to $0 \mathrm{eV}$ though it is noted that the transverse electron temperature is $2 \mathrm{meV}$. In a similar way to the DCT experiment, the product $\mathrm{O}$ atoms from the $\mathrm{DR}$ reaction were recorded with a position-sensitive detector and their KER determined on an event-by-event basis. However, in contrast to the DCT experiment, only the position of the fragments were determined and not their difference in arrival time. Hence the measured distance spectra are a superposition of two-dimensional (2D) distance distributions for each vibrational level present in the ion beam, weighted by their relative partial cross sections and branching fractions. The shape of the distance distributions and the analytical model for their superposition is described in detail in the literature..$^{20,55}$

The background contribution was again measured separately for each of the ion-source settings. For these measurements the electrons were turned off and the obtained data again subtracted. Data from both the DR and the background reactions were collected during the full storage time. In addition, each event was labeled with a time stamp relative to the start of a measuring cycle in time increments of $50 \mathrm{~ms}$.

In fitting the distance spectra, it has been assumed that the DR reaction is isotropic. Anisotropies have been observed in DR reactions, but these have only been seen at elevated collision energies and never at $0 \mathrm{eV}$ collisions. The dissociation limit given in Eq. (1d) has not been included in the fits for the reasons mentioned earlier (see Sec. I). The fitting procedure generates a distance distribution for each $\mathrm{O}_{2}^{+}(v)$ state dissociating into the four energetically possible dissociation limits, while taking into account a $300 \mathrm{~K}$ rotational distribution, the finite interaction length, and the projection of the total distance onto the $2 \mathrm{D}$ detector. The free parameters in the fit are the relative partial cross section and branching fractions of each vibrational state. These parameters are assumed to be independent of the rotational state. Only vibrational levels up to $v=5$ were taken into consideration since the higher vibrational levels were only very weakly populated and are assumed not to contribute significantly (see Sec. III A). The metastable $a^{4} \Pi_{u}$ state, when

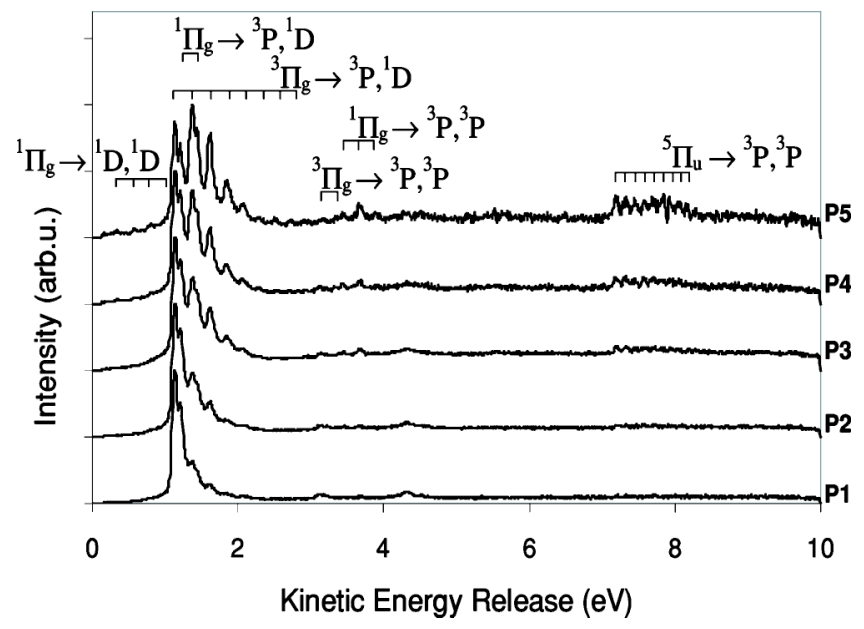

FIG. 4. Measured spectra from the $\mathrm{Cs}-\mathrm{O}_{2}^{+}$DCT reaction for the five different ion-source settings (equivalently the five vibrational populations), P1-P5.

present, will decay and produce a time varying effect on the vibrational population. Its radiative lifetime is short, most likely less than $100 \mathrm{~ms} .{ }^{56-59}$ This state has however not been included, since the decay of the metastable ions will produce an a priori unknown change in the vibrational distribution of the electronically ground-state oxygen ions. Considering arguments based only on the Franck-Condon overlap factors it is not unlikely that these product states are very highly excited ions. An additional time dependent behavior was anticipated due to the possible presence of vibrational cooling related to SEC processes. In order to investigate both of these time-dependent effects, complete analysis has been performed for data obtained during two storage-times, namely $2.5-4.0 \mathrm{~s}$ and 5.5-7.0 s. As is discussed later, there are indications that some changes occur in the first few seconds of the ring cycle, which may reduce the effect of the decaying metastable ion state after five seconds (see Sec. III C). Conversely, SECs may constantly reduce the vibrational excitation of the population with respect to the calibrated populations, which would favor the shortest possible storage time. By analyzing both sets of data, these systematic uncertainties have been transformed into uncertainties in the vibrational dependent rates and branching behavior.

\section{RESULTS}

\section{A. Vibrational populations}

The DCT spectra corresponding to the five characterized ion-source settings are shown in Fig. 4. DCT signals are observed with KERs ranging between 0 and $9 \mathrm{eV}$. The main contribution comes from dissociation events towards the $\mathrm{O}\left({ }^{3} P\right)+\mathrm{O}\left({ }^{1} D\right)$ channel [see Eq. (2a)] starting from the ${ }^{3} \Pi_{g}$ state. The associated KERs start at about $1 \mathrm{eV}$ for $\mathrm{O}_{2}^{+}\left(X^{2} \Pi_{g}, v=0\right)$ and increase with each vibrational level. Near $3 \mathrm{eV}$, events are observed corresponding to the dissociation towards the $\mathrm{O}\left({ }^{3} P\right)+\mathrm{O}\left({ }^{3} P\right)$ limit [see Eq. (2b)], which show the same vibrational structure. The small signals below $1 \mathrm{eV}$ are due to dissociation into $\mathrm{O}\left({ }^{1} D\right)+\mathrm{O}\left({ }^{1} D\right)$ [see Eq. (2c)] from $v^{\prime} \geqslant 4$. For KERs of $\sim 7 \mathrm{eV}$ and higher, DCT 
TABLE I. The populations (\%) of the vibrational levels $\mathrm{O}_{2}^{+}\left(X^{2} \Pi_{g}, v\right.$ $=0-5)$ together with the ratio of the metastable $\mathrm{O}_{2}^{+}\left(a^{4} \Pi_{u}\right)$ state to ground $\mathrm{O}_{2}^{+}\left(X^{2} \Pi_{g}\right)$ state oxygen ions, for the vibrational populations, P1-P5.

\begin{tabular}{cccccc}
\hline \hline$v$ & $\mathrm{P} 1$ & $\mathrm{P} 2$ & $\mathrm{P} 3$ & $\mathrm{P} 4$ & $\mathrm{P} 5$ \\
\hline 0 & 71 & 60 & 47 & 43 & 29 \\
1 & 16 & 22 & 26 & 29 & 30 \\
2 & 7 & 9 & 14 & 16 & 22 \\
3 & 4 & 5 & 8 & 9 & 10 \\
4 & 2 & 3 & 4 & 4 & 5 \\
5 & 1 & 1 & 2 & 1 & 4 \\
$a^{4} \Pi_{u} / X^{2} \Pi_{g}$ & 0.01 & 0.05 & 0.11 & 0.14 & 0.29 \\
\hline \hline
\end{tabular}

signals arising from the metastable $a^{4} \Pi_{u}$ state are also observed [see Eq. (3)]. Consideration of the complete spectrum unambiguously reveals the vibrational population of the ion beam. All five ion-source settings were chosen such that the vibrational excitation of the ions gradually increased. The label P5 refers to the most excited population. Here, the $v$ $=1$ population exceeds the $v=0$ population and a significant fraction of metastable $a^{4} \Pi_{u}$ oxygen ions are observed. The DCT spectrum of P5 reveals the nascent population created by $100 \mathrm{eV}$ electron impact ionization. It is noted that the coldest population, referred to as $\mathrm{P} 1$, does not contain $v=0$ ions only. Table I lists the derived vibrational populations for the levels $v=0-5$ together with the ratio between the ground and the metastable state. More details of the interpretation of the DCT dynamics can be found in literature. ${ }^{43}$

\section{B. Dissociative recombination rates and branching fractions}

\section{Total rate coefficients}

The measured total rate coefficients for the $\mathrm{DR}$ of $\mathrm{O}_{2}^{+}$in each of the five vibrational populations, P1-P5, have been obtained for electron energies up to $0.4 \mathrm{eV}$. The results are shown in Fig. 5(a). Though error bars are not shown, the main source of error in the relative data is statistical. Near 0 $\mathrm{eV}$ this error is smaller than the separation between the curves, while above $100 \mathrm{meV}$ the statistical error starts to become significant. A systematic uncertainty in the absolute data arises from the measurement of the ion current, and is estimated ${ }^{13}$ to be around $20 \%$. We observe that the total rate coefficient decreases upon increasing vibrational excitation near $0 \mathrm{eV}$ collision energy. This observation suggests that the partial cross section for $v=0$ is higher than that for $v=1$. The total rate coefficient of the hottest population, $k_{\mathrm{P} 5}$, is, in general, the slowest though for collision energies between 210 and $330 \mathrm{meV}$ this rate is as fast as those of the less excited populations. The observed rates vary much less than an order of magnitude. The corresponding thermal rate coefficients, $\alpha\left(\mathrm{O}_{2}^{+}\right)$, can be derived from the total measured rates by determining the thermal average weighted by the thermal electron distribution. The thermal rate coefficients at $300 \mathrm{~K}$ are calculated to be $(2.0,1.9,1.9,1.8$, and 1.5$) \cdot 10^{-7} \mathrm{~cm}^{3} \mathrm{~s}^{-1}$ for P1-P5, respectively. The small variation is consistent with the small range of thermal rates previously reported in literature (see Sec. I).

The general behavior of the rate coefficient due to the direct DR process is proportional to the inverse square root
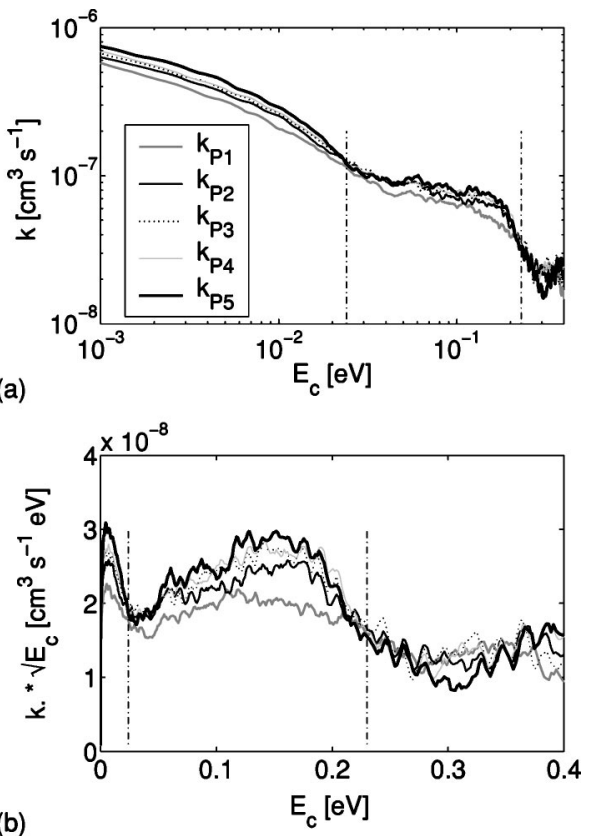

FIG. 5. (a) The total rate coefficients, $k_{\mathrm{P} i}$, as function of collision energy, $E_{c}$, up to $0.4 \mathrm{eV}$ where $\mathrm{P} i=\mathrm{P} 1-\mathrm{P} 5$, on a bi-logarithmic scale, (b) and on a bilinear scale when multiplied by the square root of the collision energy, revealing the resonances due to nondirect dissociation mechanisms. The dashed vertical lines at 0.024 and $0.23 \mathrm{eV}$ indicate the energy of the spinorbit splitting in the $X^{2} \Pi_{g},(\Omega=1 / 2,3 / 2)$ states and the vibrational spacing, respectively.

of the collision energy (see Sec. I). Therefore, multiplying the rate by the square root of the collision energy will remove the $1 / \sqrt{E_{c}}$ dependency and resonances in the DR process will appear as deviations around a collision-energyindependent level. These data can then be plotted on a linear scale [see Fig. 5(b)]. The total rate coefficient of the coolest population, $k_{\mathrm{P} 1}$, shows strong deviations from the purely $1 / \sqrt{E_{c}}$ behavior. Upon increasing excitation, the total rate coefficient becomes flatter and the variations are virtually absent for the hottest population P5. Clearly the different vibrational states have a qualitatively different behavior. In the following section the total rate coefficients are used as a consistency check on the partial rate coefficients as derived from the imaging experiment.

\section{Partial rate coefficients at $0 \mathrm{eV}$}

Each of the five vibrational populations will produce a different distance distribution spectrum if the partial cross sections and branching fractions have a vibrational dependence. Figure 6 shows some of these measured distance distributions obtained for $0 \mathrm{eV}$ collisions. The solid curves show the fits, which combine a series of known instrument parameters and, as free parameters, the relative partial cross section and branching fractions over the four dissociation limits for each vibrational level of the parent ion. As mentioned before, data have been obtained and analyzed from two different storage times, 2.5-4.0 s and 5.5-7.0 s, respectively. The spectra for population P1 are shown in Figs. 6(a) and 6(b) and for population P5 in Figs. 6(c) and 6(d). The $x$-axes are the projected distances between the two $\mathrm{O}$ product 
(a)

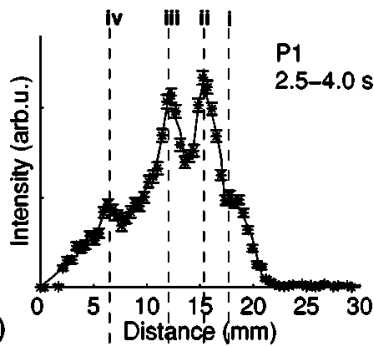

(c)

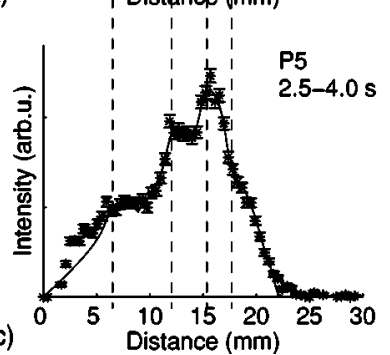

(b)

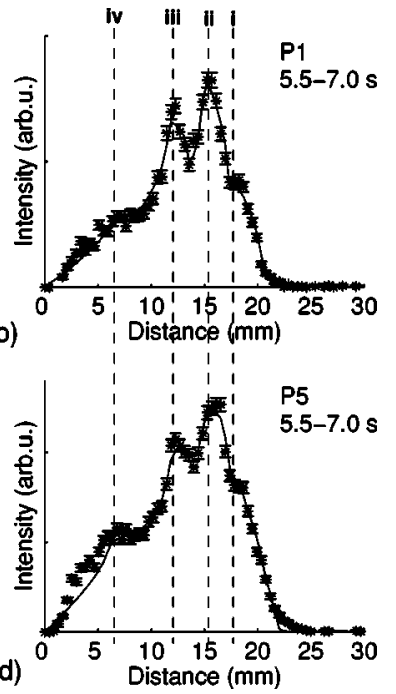

FIG. 6. The distance distributions (stars) and their fits (black solid curves) normalized to unit area, of population P1, obtained during storage times (a) 2.5-4.0 s and (b) 5.5-7.0 s and of population P5, obtained during storage times (c) 2.5-4.0 s and (d) 5.5-7.0 s. The dashed lines i-iv indicate the four dissociation limits given in Eqs. (1a)-(1c) and (1e), respectively. The distance distributions are in fact the histograms of all the projected distances between the two $\mathrm{O}$ product atoms.

atoms, which is roughly proportional to the square root of the $\mathrm{KER}^{20}$ The four dissociation limits can easily be distinguished (peaks i-iv). The spectrum for P1 differs markedly from the spectrum for P5, indicating a vibrational dependence of the dissociation reaction. The width of the peaks is determined by the level of vibrational excitation present. Higher vibrational states give rise to higher KERs and thus broaden the electronic peaks to larger distances. The effect of vibrational excitation is most apparent in the $\mathrm{O}\left({ }^{1} S\right)$ dissociation (peak iv), since there the vibrational energy spacing is of the order of the KER, $0.8 \mathrm{eV}$. Analysis of the peak-width shows that P1 is vibrationally more relaxed than P5. Due to the method of data acquisition, the absolute number of counts cannot be used to estimate the relative total rate coefficients for the different populations.

The spectra also differ in more subtle ways. The P5 distance distributions show a small tail at large distances, i.e., at higher KERs, which cannot be fitted when including only the first 6 vibrational levels. This tail is also observed in the distance distributions obtained from the populations P3 and P4 (not shown), but is not seen in the P1 and P2 distributions ( $\mathrm{P} 2$ also not shown). This is consistent with the production of highly vibrationally excited $\mathrm{O}_{2}^{+}$ions resulting from the decay of the first electronically excited metastable $a^{4} \Pi_{u}$ state in P3-P5 (see Fig. 4). Furthermore, signals are observed at very small KERs (distances below $6 \mathrm{~mm}$ ) in the P5 spectra, which are not present in the P1 spectra. This may indicate the opening of the next highest fragmentation channel, which can only occur for vibrational levels higher than $v=7$ [see Eq. (1f)]. Finally, when comparing P1-P5, it is noted that the dissociation peaks in the P5 spectra are not only broader, but have also moved slightly to larger distances, i.e., higher KER, reflecting the shift in vibrational population.

The distance distributions from P2-P5 were fitted with
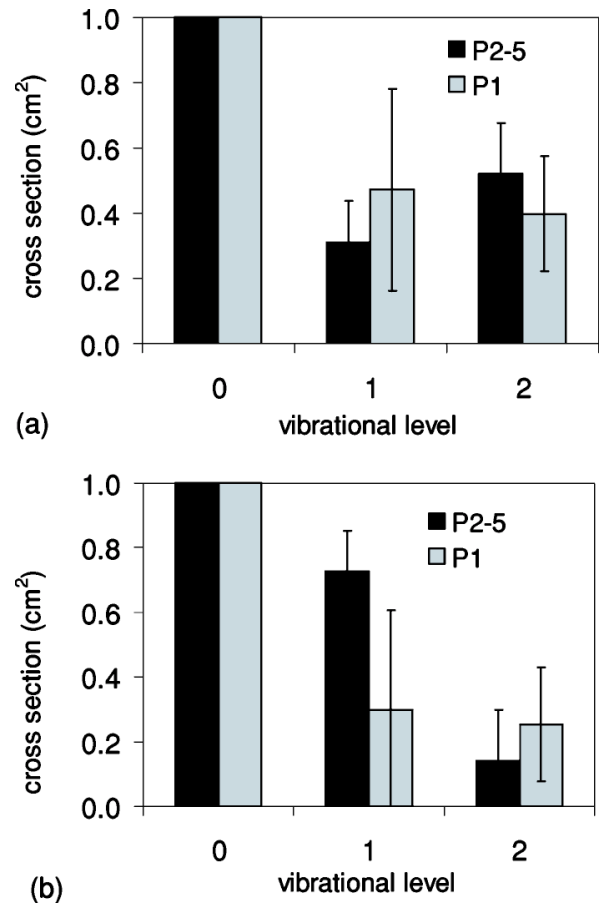

FIG. 7. The partial cross sections, $\sigma_{v}$, for $v=0-2$, relative to $\sigma_{0}$, at $0 \mathrm{eV}$ collision energy resulting from the simultaneous fit of P2-P5 data (black) and the separate fit of P1 data (gray) obtained during storage times (a) 2.5-4.0 s and (b) $5.5-7.0 \mathrm{~s}$.

$v=0-5$, while simultaneously optimizing the partial cross section and branching fractions for each vibrational level. We have fitted the distance distributions from P1 separately, only considering the first four vibrational levels, $v=0-3$. The latter fit serves as a consistency check for the simultaneous fitting of P2-P5. Since neither the product vibrational states created from the decaying metastable state nor the vibrational cooling effect of the SEC processes are quantitatively included in the fitting procedure, systematic errors are introduced, especially for the sparsely populated higher vibrational levels. We, therefore, present the vibrationally resolved results for the vibrational levels $v=0,1$, and 2 only.

The relative partial cross sections, $\sigma_{v}$, obtained for $v$ $=0-2$ during these two storage times are given in Fig. 7 . The data are plotted relative to the cross section obtained for $v=0$. It is observed that the cross section calculated for $v$ $=0$ is the highest. The cross sections obtained from the separate fit for P1 and the simultaneous fit for P2-P5 are consistent for the data taken during the storage time $2.5-4.0 \mathrm{~s}$. The results obtained during the storage time $5.5-7.0 \mathrm{~s}$ are different, although not significantly. The cross section obtained for $v=1$ from the fit to P1 data appears to be much lower. When comparing the data obtained during the two storage times to each other, the cross sections differ significantly. For the shorter storage time, the rate obtained for $v=1$ is much lower than that calculated from the data obtained at longer storage time. The opposite is true for $v=2$. The disagreement suggests that the vibrational population in the ion beam was different for the different storage times.

A second consistency test for the obtained results is the reconstruction of relative total cross sections, $\sigma_{\mathrm{P} i}^{\prime}$, for $\mathrm{P} i$ $=\mathrm{P} 1-\mathrm{P} 5$ based on the partial cross sections, $\sigma_{v}$, and use 
TABLE II. The relative total cross sections $\sigma_{\mathrm{P} i}$ deduced from the measured total rate coefficients together with the reconstructed relative total cross sections $\sigma_{\mathrm{P} i}^{\prime}$ using Eq. (4) with $\sigma_{v}$ the partial cross sections from the simultaneous fit at 2.5-4.0 s and 5.5-7.0 s storage time, respectively.

\begin{tabular}{lcccccc}
\hline \hline & & $\mathrm{P} 1$ & $\mathrm{P} 2$ & $\mathrm{P} 3$ & $\mathrm{P} 4$ & $\mathrm{P} 5$ \\
\hline$\sigma_{\mathrm{P} i}$ & & 1.13 & 1.02 & 1.00 & 0.95 & 0.83 \\
$\sigma_{\mathrm{P} i}^{\prime}$ & $2.5-4.0 \mathrm{~s}$ & 1.21 & 1.11 & 1.00 & 0.95 & 0.84 \\
& $5.5-7.0 \mathrm{~s}$ & 1.19 & 1.11 & 1.00 & 0.96 & 0.83 \\
\hline \hline
\end{tabular}

these reconstructed total cross sections to compare with the relative total cross sections, $\sigma_{\mathrm{P} i}$, calculated from the five measured total rate coefficients, $k_{\mathrm{P} i}$, (see Fig. 5) near $0 \mathrm{eV}$ collision energy:

$$
\sigma_{\mathrm{P} i}^{\prime}=\sum_{v=0}^{2} \sigma_{v} \mathrm{Pi}(v) \quad \text { for } i=1 \ldots 5 \text {. }
$$

The outcome of this test is given in Table II. The reconstructed data is remarkably consistent with the measured total cross sections. The qualitative conclusion that the measured total rates can be understood by assuming a faster DR rate for $v=0$ is corroborated quantitatively.

\section{Partial branching fractions at $0 \mathrm{eV}$}

Analysis of the vibrationally dependent partial rate coefficients also provides their branching behavior. Figure 8 shows the partial branching fractions for the levels $v$ $=0-2$. Results are again presented based on the simultaneous fit of P2-P5 data and the separate fit of P1 data for the two investigated storage times, $2.5-4.0 \mathrm{~s}$ and $5.5-7.0 \mathrm{~s}$, respectively. Table III lists the resulting partial branching fractions together with the partial cross sections as determined by the simultaneous fit at $2.5-4.0 \mathrm{~s}$ together with the vibrationally state dependent quantum yields for $\mathrm{O}\left({ }^{1} S\right), \mathrm{O}\left({ }^{1} D\right)$, and $\mathrm{O}\left({ }^{3} P\right)$. These quantum yields are easily derived from the partial branching fractions. The yields are the relevant observables for in situ atmospheric observations and also in afterglow or discharge type experiments. From both Fig. 8 and Table III it is concluded that the partial branching fractions are strongly dependent on the vibrational state. The $\mathrm{O}\left({ }^{1} S\right)$ quantum yield increases upon vibrational excitation, and by more than a factor of three in going from $v=0$ to $v=2$. The $\mathrm{O}\left({ }^{1} D\right)$ quantum yield is lowest at $v=0$, whereas the $\mathrm{O}\left({ }^{3} P\right)$ yield is highest at $v=0$.


FIG. 8. The partial branching fractions for $\mathrm{O}_{2}^{+}\left(X^{2} \Pi_{g}, v=0-2\right)$ towards the 4 dissociation limits given in Eqs. (1a)-(1c) and (1e) for the simultaneous fit of P2-P5 data (first column of each column pair) and the separate fit of P1 data (second column of each column pair) obtained during storage times (a) $2.5-4.0 \mathrm{~s}$ and (b) $5.5-7.0 \mathrm{~s}$.

\section{Time-dependent data}

\section{Vibrational cooling}

In an ion storage ring experiment, the ion beam population can change due to a one of several mechanisms. Ignoring DR for the moment, the main destruction of the ion beam arises from collisions with rest gas molecules inside the ring. The efficiency of these collisions may be dependent on the vibrational state. However, we observe no changes in the ion-beam lifetime upon changing vibrational populations (electrons off). Although we have indications that the product distribution due to charge transfer induced dissociation depends on the vibrational state in the ion beam (see below), we have no information on the associated partial rates. Under very good vacuum conditions, which is the case in most heavy ion storage rings, the DR process in the electron cooler may be so efficient as to radically deplete certain states from the ion beam. This is observed ${ }^{55}$ to be the case for the DR of $\mathrm{H}_{2}^{+}$. In the current experiment, the lifetime of the ion beam is not significantly affected when the electrons are turned on. Thus, the loss of ions due to the DR process is too small to compete with that due to collisions with rest gas. This leaves the SEC process as a possible mechanism to change the vibrational population of the ion-beam via a se-

TABLE III. The partial cross sections, quantum yields and branching fractions for $\mathrm{O}_{2}^{+}\left(X^{2} \Pi_{g}, v=0-2\right)$ resulting from the simultaneous fit of the P2-P5 data obtained during storage time $2.5-4.0 \mathrm{~s}$.

\begin{tabular}{|c|c|c|c|c|c|c|c|c|}
\hline \multirow[b]{2}{*}{$v$} & \multirow[b]{2}{*}{$\sigma_{v}$} & \multicolumn{3}{|c|}{ Quantum yields } & \multicolumn{4}{|c|}{ Branching fractions } \\
\hline & & $\mathrm{O}\left({ }^{1} S\right)$ & $\mathrm{O}\left({ }^{1} D\right)$ & $\mathrm{O}\left({ }^{3} P\right)$ & $\mathrm{O}\left({ }^{1} D\right)+\mathrm{O}\left({ }^{1} S\right)$ & $\mathrm{O}\left({ }^{1} D\right)+\mathrm{O}\left({ }^{1} D\right)$ & $\mathrm{O}\left({ }^{3} P\right)+\mathrm{O}\left({ }^{1} D\right)$ & $\mathrm{O}\left({ }^{3} P\right)+\mathrm{O}\left({ }^{3} P\right)$ \\
\hline 0 & 1 & 0.06 & 0.94 & 1.00 & $5.8 \pm 0.5$ & $20.4 \pm 0.3$ & $47.3 \pm 0.8$ & $26.5 \pm 0.8$ \\
\hline 1 & $0.31 \pm 0.13$ & 0.14 & 1.44 & 0.42 & $13.9 \pm 3.1$ & $51.0 \pm 5.4$ & $27.8 \pm 5.1$ & $7.3 \pm 7.5$ \\
\hline 2 & $0.52 \pm 0.16$ & 0.21 & 1.02 & 0.76 & $21.1 \pm 2.5$ & $2.5 \pm 2.1$ & $76.4 \pm 2.2$ & $0.02 \pm 0.03$ \\
\hline
\end{tabular}





FIG. 9. (a) The distance distributions of P5 obtained during 2.5-4.0 s (black circles) and $8.5-10.0 \mathrm{~s}$ (gray circles) storage time and (b) the distance distributions of P5 obtained during 8.5-10.0 s (gray circles) and of P4 obtained during $2.5-4.0 \mathrm{~s}$ (black squares) storage time. Note that all distributions are normalized to their own maximum.

ries of $\Delta v=-1$ transitions. This mechanism has been established in various experiments ${ }^{50,51}$ on $_{2}^{+}$. In the current experiment, time dependent behavior of the ion beam is observed indicating changes in the vibrational population. It is concluded that the SEC process is more efficient than the DR process in changing the vibrational population of the stored ion beam.

To investigate the efficiency of the SEC process in changing the characterized populations, imaging data were obtained during storage times up to $10 \mathrm{~s}$. Figure 6 already shows the distance distributions of both P1 and P5 obtained during the two storage times $2.5-4.0 \mathrm{~s}$ and $5.5-7.0 \mathrm{~s}$ (see Sec. III B). For the P5 data, neither the widths of the observed peaks narrow significantly nor do the highest KERs (at 23-25 mm) disappear. The only indication of a changing vibrational population is the change in the relative amplitudes of the peaks. Figure 9(a) plots the distance distribution from the P5 data obtained during 2.5-4.0 s together with the spectrum obtained during even longer storage time, 8.5-10.0 s. The $\mathrm{O}\left({ }^{1} S\right)$ peak (at $\sim 7 \mathrm{~mm}$ ) has narrowed, indicating cooling has occurred. However, the high KER tail at 23-25 $\mathrm{mm}$ still shows no change. Figure 9(b) compares the data from P5 obtained at 8.5-10.0 s with that obtained from the somewhat less excited population P4 at 2.5-4.0 s. Analysis of these data suggests that it takes more than $8 \mathrm{~s}$ for the higher vibrationally excited population to cool down to the less excited population. The cooling mechanisms inside the ring seem to be less effective than those operating in the ion source.

\section{Background dynamics}

Background imaging data taken for all populations $\mathrm{P} 1-\mathrm{P} 5$ give some evidence of vibrational dependence in the dynamics of the charge transfer process between the $\mathrm{O}_{2}^{+}$ions and the residual gas molecules in the ring. The 2-particle events resulting from these reactions give rise to distance distributions that can be related to the ion-beam dynamics similar to the DR spectra. Here the interaction region is not determined by the length of the electron cooler but by the entire straight section. The associated distance distributions reveal different dissociation dynamics for the excited population P5 at 2.5-4.0 s storage time, compared to the other data. Such differences are no longer observed at 5.5-7.0 s storage time and, for all other populations, the charge transfer distance spectra were found to be almost identical. The main difference is observed at larger KERs. To investigate this, attempts were made to reduce the metastable fraction in the ion beam by decreasing the electron impact energy in the ion source from 100 to $23 \mathrm{eV}$. At this energy the vibrational excitation of the ground state remains approximately the same. Although the data obtained under these conditions still showed differences at larger distances, their lower magnitude suggests that the product vibrational levels resulting from the decaying metastable state are at least partly responsible. The energy of these vibrational levels in the DR distance spectrum is unknown, though they must be different from those responsible for the signal seen at 23-25 mm (see Fig. 6) for the P3-P5 settings, since this tail did not disappear in the DR spectrum of P5 after $5.5 \mathrm{~s}$ of cooling.

\section{DISCUSSION}

In laboratory experiments the vibrational dependence of the DR reaction is difficult to study in a well-controlled way. ${ }^{12,20,34,60}$ In the research presented here we have managed to control and reproduce a number of significantly different vibrational populations. We were able to smoothly vary the ion population from vibrationally cool to vibrationally hot. This has allowed us to determine the vibrational dependence of the DR cross sections and branching fractions for the first few vibrational levels. The present experiment is limited by two factors. The first is the finite resolution of the imaging method, since the absence of vibrational resolution increases the uncertainty in the fitting procedure. A better spatial resolution or maybe even arrival-time information of the product atoms will substantially improve the results. The second is related to time-dependent changes of the vibrational populations in the ion beam during its storage time. The lowest lying metastable $a^{4} \Pi_{u}$ state is formed efficiently in the source and decays down to the ground state with some unknown vibrational population. Furthermore, inelastic electron scattering, in SEC processes, also induce some changes in the vibrational population. In spite of these effects, we believe that the estimates given here for the vibrationally dependent rates and branching behavior are the most accurate laboratory estimates to date.

The observed total $\mathrm{O}_{2}^{+}$DR rate coefficient as function of collision energy and vibrational population were relatively straightforward to analyze and these data are the most reliable. The observations show that the total DR rate coefficient depends weakly on the vibrational population of the parent ion beam. For low collision energies the rate decreases with 
increasing vibrational excitation. This decrease is less than a factor of 2 and excludes vibrational specific rates that differ by an order of magnitude, as is found ${ }^{61}$ for $\mathrm{HD}^{+}$. The calculated thermal rate coefficient, $\alpha\left(\mathrm{O}_{2}^{+}\right)$, at $300 \mathrm{~K}$ corresponding to the coolest population $\mathrm{P} 1$ is $2.0 \cdot 10^{-7} \mathrm{~cm}^{3} \mathrm{~s}^{-1}$ and decreases to $1.5 \cdot 10^{-7} \mathrm{~cm}^{3} \mathrm{~s}^{-1}$ for the hottest population P5. The small range is consistent with the small range of previously reported thermal rate coefficients (see Sec. I). The total rate coefficient curves also show variations from the general behavior $k\left(\mathrm{O}_{2}^{+}\right) \sim E_{c}^{-0.5}$. These variations are due to resonances, which differ from the general behavior arising from direct capture-dissociation mechanisms. The resonance structure decreases with increasing vibrational population, being most strong in the coolest population and almost nonexistent in the most excited population. Some characteristic energy signatures in the $\mathrm{O}_{2}^{+}$ion coincide with features in these observed resonances (see Fig. 5). The spin-orbit splitting in the $X^{2} \Pi_{g},(\Omega=1 / 2,3 / 2)$ states of $197 \mathrm{~cm}^{-1}(\sim 24$ $\mathrm{meV}$ ) and the vibrational spacing of $0.23 \mathrm{eV}$ both coincide with collision energies where the calculated total rate coefficients for the different vibrational populations are almost identical and which then all decrease towards a local minimum.

The interpretation of the imaging data at $0 \mathrm{eV}$ collisions is less trivial, however, the effect of the super elastic collisions and the presence of the metastable state have been qualitatively studied by obtaining data at two different storage times. Additionally, the sensitivity of the fitting procedure has been investigated and based on this we have restricted our findings to the lowest three vibrational levels. We find that both the partial cross sections and branching fractions depend on the vibrational level. The partial rate coefficients are found to be consistent with the observed total rate coefficients at zero eV collision energy. The partial cross section is the fastest for $v=0$ and decreases by a factor of more than 2 for $v=1$ and 2. The $\mathrm{O}\left({ }^{1} D\right)$ quantum yield is lowest, and the $\mathrm{O}\left({ }^{3} P\right)$ yield highest, at $v=0$. The $\mathrm{O}\left({ }^{1} S\right)$ quantum yield increases by more than a factor of 2 for $v$ $=1$ and 2 . Our results display a very strong dependence on the vibrational level.

\section{A. $\mathrm{O}_{2}^{+}$and similar systems}

In the case of $\mathrm{O}_{2}^{+}$only a limited number of experiments exist that report any vibrational state-dependent behavior, and these are mostly rate coefficient data. Kella et $a .^{20}$ measured the DR branching behavior of an unknown vibrational population of the mixed isotopomer ${ }^{16} \mathrm{O}^{18} \mathrm{O}^{+}$. In Kella et al.'s study, only the total quantum yields summed over all the vibrational levels are reported. The total $\mathrm{O}\left({ }^{1} S\right)$ yield for the vibrationally excited ions is reported to be a factor of 2 larger than the $\mathrm{O}\left({ }^{1} S\right)$ yield for ground state ions. The total $\mathrm{O}\left({ }^{1} D\right)$ and $\mathrm{O}\left({ }^{3} P\right)$ yields are reported to show no significant vibrational dependence. One further paper reports results on the branching behavior of $\mathrm{O}_{2}^{+}\left(X^{2} \Pi_{g}, v=0\right)$ ions. ${ }^{13}$ The results reported in this paper agree in the most part with those presented here. It is noted that their $\mathrm{O}\left({ }^{1} D\right)$ quantum yield is higher than the $\mathrm{O}\left({ }^{3} P\right)$ yield, whereas the reverse is true in our case. The reason for this discrepancy is not clear at present. The current results for the ground vibrational level of $\mathrm{O}_{2}^{+}$are consistent with data obtained from an independent experiment performed on the collision-energy dependence of the DR reaction. ${ }^{24}$

It has been suggested that the DR process is very similar for small diatomic ions, i.e., predominantly direct dissociation, giving the equivalent order and temperature dependence of the thermal rate coefficient. In most of these systems we only have branching data for ground-state ions, for examples $\mathrm{NO}^{+}$and $\mathrm{CO}^{+}$. This may be because these ions easily relax radiatively to their ground state. Furthermore, little is known on the vibrational behavior of their DR rate coefficients. However, a few measurements on the total rate coefficient of $\mathrm{CO}^{+}$show a higher rate for vibrational ground-state ions ${ }^{62,63}$ than for vibrationally excited ions. ${ }^{64}$ For $\mathrm{NO}^{+}$, the measured thermal rate of vibrationally excited ions is also reported to be lower than that of vibrational ground-state ions. ${ }^{65}$ In the case of $\mathrm{N}_{2}^{+}$an experiment performed at CRYRING reported a weakly dependent total rate coefficient, where the rate of the vibrationally excited beam was again lower than that of the ground state. ${ }^{66}$ In this paper there is also mention of the branching fractions of the $v=1-3$ vibrational levels being roughly the same. Finally, a recently published review reports that the recombination rates measured for vibrationally excited $\mathrm{N}_{2}^{+}, \mathrm{O}_{2}^{+}$, and $\mathrm{NO}^{+}$are lower than their equivalent ground-state ions. ${ }^{67}$ Specifically, they report that the difference is about a factor of 2 in the case of $\mathrm{O}_{2}^{+}$over the temperature range $200-4000 \mathrm{~K}$.

\section{B. Theory and modeling}

The issue of vibrationally dependent DR rates and branching fractions (and associated fragment quantum yields) has given rise to extensive and often inconclusive discussions in literature. Vibrationally dependent DR behavior is often inferred from ionospheric observations, ${ }^{31,32,68-70}$ whereas studies on the production and quenching of $\mathrm{O}_{2}^{+}(v)$ in the ionosphere often conclude the vibrational deactivation is fast. ${ }^{9,35,37}$ Fox shows, however, that above $200 \mathrm{~km}$ the $\mathrm{O}_{2}^{+}$ vibrational population can already be significant. ${ }^{71}$ The present results, combined with Fox's populations, could already be used in modeling of the increasing green line emission upon altitude. However, energetic information on the partial rates and yields is still needed for a precise comparison with atmospheric observation.

To the best of our knowledge no vibrational dependent branching has been predicted from purely $a b$ initio calculations. The earlier work of Guberman identified a number of possible target states in the DR process though the complexity of molecular oxygen makes it difficult to draw general conclusions on the process. ${ }^{22}$ As has been mentioned earlier, molecular oxygen is somewhat special due to the very large avoided crossings between the various repulsive molecular states and the lowest $(n=3$ and $n=4)$ Rydberg states (see Fig. 1). As a consequence, there are potential barriers on the exit channels leading to the lower-lying dissociation limits. At most avoided crossings the dissociation flux tries to keep some Rydberg character. Furthermore, molecular oxygen is the first system in which a spin-orbit mechanism has been invoked to describe the production of $\mathrm{O}\left({ }^{1} S\right)$ atoms near $0 \mathrm{eV}$ 
collisions for $v=0$ ions. A resonance near $0 \mathrm{eV}$, which causes the colliding electron to be captured in a vibrationally excited Rydberg state, creates a sufficient time delay such that coupling of the ${ }^{3} \Sigma_{u}^{-}$and the ${ }^{1} \Sigma_{u}^{+}$becomes effective in redirecting flux over the repulsive ${ }^{1} \Sigma_{u}^{+}$valence state towards the $\mathrm{O}\left({ }^{1} S\right)+\mathrm{O}\left({ }^{1} D\right)$ limit, explaining the experimentally observed finite quantum yield.

\section{CONCLUSIONS}

In summary, the present research details considerable progress in the control of vibrational populations and has provided more valuable insight into the vibrational dependence of the DR of $\mathrm{O}_{2}^{+}$. Partial cross sections, branching fractions and quantum yields for $v=0-2$ together with total rate coefficients for five different vibrational populations have been obtained and are reported here. The partial branching fractions and quantum yields together with the partial cross sections are strongly dependent on the vibrational level of the parent ion, with the yield of $\mathrm{O}\left({ }^{1} S\right)$ increasing substantially upon increasing vibrational level. The partial cross sections agree with the measured total cross sections. The weak dependence of the total rate coefficients on the vibrational population is supported by the small range of thermal rates previously reported. Additionally, the decrease in total rate upon excitation seems to be in agreement with similar systems. The present results may be extended to higher vibrational levels once the difficulties in the experimental and analytical approaches have been overcome.

\section{ACKNOWLEDGMENTS}

This work is part of the research program of the "Stichting voor Fundamenteel Onderzoek der Materie (FOM)," which is financially supported by the "Nederlandse organisatie voor Wetenschappelijk Onderzoek (NWO)." It has been partially supported by the NASA Planetary Atmospheres Program under Grant No. NAG5-11173 to SRI International. Support has also been given by the EU research-training network Electron Transfer Reactions (ETR) under HPRN-CT2000-00142. We thank the staff of the Manne Siegbahn Laboratory for their part in making the experiments possible.

${ }^{1}$ J. L. Fox, J. Geophys. Res. 102, 24005 (1997).

${ }^{2}$ R. R. Hodges, Jr., J. Geophys. Res. 105, 6971 (2000).

${ }^{3}$ M. A. Biondi and S. C. Brown, Phys. Rev. 76, 1697 (1949).

${ }^{4}$ M. A. Biondi, Ann. Geophys. (C.N.R.S.) 20, 34 (1964).

${ }^{5}$ W. H. Kasner and M. A. Biondi, Phys. Rev. 174, 139 (1968).

${ }^{6}$ F. J. Mehr and M. A. Biondi, Phys. Rev. 181, 264 (1969).

${ }^{7}$ D. Smith, C. V. Goodall, N. G. Adams, and A. G. Dean, J. Phys. B 3, 34 (1970).

${ }^{8}$ A. J. Cunningham and R. M. Hobson, J. Phys. B 5, 2320 (1972).

${ }^{9}$ E. C. Zipf, J. Geophys. Res. 85, 4232 (1980).

${ }^{10}$ E. Alge, N. G. Adams, and D. Smith, J. Phys. B 16, 1433 (1983).

${ }^{11}$ P. Š Spanĕl, L. Dittrichová, and D. Smith, Int. J. Mass Spectrom. Ion Processes 129, 183 (1993).

${ }^{12}$ T. Gougousi, M. F. Golde, and R. Johnsen, Chem. Phys. Lett. 265, 399 (1997).

${ }^{13}$ R. Peverall et al., J. Chem. Phys. 114, 6679 (2001).

${ }^{14}$ F. L. Walls and G. H. Dunn, J. Geophys. Res. 79, 1911 (1974).

${ }^{15}$ J. W. McGowan et al., Phys. Rev. Lett. 42, 373 (1979).

${ }^{16}$ P. M. Mul and J. W. McGowan, J. Phys. B 12, 1591 (1979).

${ }^{17}$ E. C. Zipf, Bull. Am. Phys. Soc. 15, 418 (1970).

${ }^{18}$ E. C. Zipf, Geophys. Res. Lett. 6, 881 (1979).
${ }^{19}$ H. Helm, I. Havell, C. W. Walter, and P. C. Cosby, in Dissociative Recombination: Theory, Experiment and Applications III, edited by D. Zajfman, J. B. A. Mitchell, D. Schwalm, and B. R. Rowe (World Scientific, Singapore, 1996), p. 139.

${ }^{20}$ D. Kella, L. Vejby-Christensen, P. J. Johnson, H. B. Pedersen, and L. H. Andersen, Science 276, 1530 (1997).

${ }^{21}$ R. Peverall et al., Geophys. Res. Lett. 27, 481 (2000).

${ }^{22}$ S. L. Guberman, Science 278, 1276 (1997).

${ }^{23}$ S. L. Guberman, Int. J. Quantum Chem. S13, 531 (1979).

${ }^{24}$ A. Petrignani et al. (unpublished).

${ }^{25}$ G. Hernandez, Planet. Space Sci. 19, 467 (1971).

${ }^{26}$ P. B. Hays and W. E. Sharp, J. Geophys. Res. 78, 1153 (1973).

${ }^{27}$ J. E. Frederick et al., J. Geophys. Res. 81, 3923 (1976).

${ }^{28}$ J. P. Kopp, J. E. Frederick, D. W. Rusch, and G. A. Victor, J. Geophys. Res. 82, 4715 (1977).

${ }^{29}$ W. E. Sharp and D. G. Torr, J. Geophys. Res. 84, 5345 (1979).

${ }^{30}$ R. R. O'Neil, E. T. P. Lee, and E. R. Huppi, J. Geophys. Res. 84, 823 (1979).

${ }^{31}$ V. J. Abreu, S. C. Solomon, W. E. Sharp, and P. B. Hays, J. Geophys. Res. 88, 4140 (1983).

${ }^{32}$ J. H. A. Sobral et al., Planet. Space Sci. 40, 607 (1992).

${ }^{33}$ D. R. Bates, Planet. Space Sci. 40, 893 (1992).

${ }^{34}$ J. L. Queffelec, B. R. Rowe, F. Vallée, J. C. Gomet, and M. Morlais, J. Chem. Phys. 91, 5335 (1989).

${ }^{35}$ D. R. Bates and E. C. Zipf, Planet. Space Sci. 28, 1081 (1980).

${ }^{36}$ M. R. Torr and D. G. Torr, Rev. Geophys. Space Phys. 20, 91 (1982).

${ }^{37}$ H. Böhringer, M. Durup-Ferguson, E. E. Ferguson, and D. W. Fahey, Planet. Space Sci. 31, 483 (1983).

${ }^{38}$ T. G. Slanger, P. C. Cosby, D. L. Huestis, and T. A. Bida, Science 291, 463 (2001).

${ }^{39}$ S. L. Guberman, in Physics of Ion-Ion and Electron-Ion Collisions, edited by F. Brouillard (Plenum, New York, 1983), p. 167.

${ }^{40}$ S. L. Guberman and A. Giusti-Suzor, J. Chem. Phys. 95, 2602 (1991).

${ }^{41}$ S. L. Guberman, J. Chem. Phys. 67, 1125 (1977).

${ }^{42}$ S. Datz et al., Phys. Rev. Lett. 85, 5555 (2000).

${ }^{43}$ C. W. Walter, P. C. Cosby, and J. R. Peterson, J. Chem. Phys. 98, 2860 (1993).

${ }^{44}$ A. B. van der Kamp, J. H. M. Beijersbergen, P. C. Cosby, and W. J. van der Zande, J. Phys. B 27, 5037 (1994).

${ }^{45}$ J. R. Peterson and Y. K. Bae, Phys. Rev. A 30, 2807 (1984).

${ }^{46}$ W. J. van der Zande, W. Koot, J. R. Peterson, and J. Los, Chem. Phys. Lett. 140, 175 (1987).

${ }^{47}$ W. J. van der Zande, W. Koot, J. R. Peterson, and J. Los, Chem. Phys. 126, 169 (1988).

${ }^{48}$ M. Larsson, Annu. Rev. Phys. Chem. 48, 151 (1997).

${ }^{49}$ F. Hellberg et al., J. Chem. Phys. 118, 6250 (2003).

${ }^{50}$ S. Krohn et al., Phys. Rev. A 62, 032713 (2000).

${ }^{51}$ D. Zajfman et al., Nucl. Instrum. Methods Phys. Res. B 205, 360 (2003).

${ }^{52}$ A. Al-Khalili et al., Phys. Rev. A 68, 042702 (2003).

${ }^{53}$ M. B. Någård et al., J. Chem. Phys. 117, 5264 (2002).

${ }^{54}$ A. Neau et al., J. Chem. Phys. 113, 1762 (2000).

${ }^{55}$ W. J. van der Zande et al., Phys. Rev. A 54, 5010 (1996).

${ }^{56}$ A. O'Keefe and J. R. McDonald, Chem. Phys. 103, 425 (1986).

${ }^{57}$ S. W. Bustamente et al., J. Chem. Phys. 86, 508 (1987).

${ }^{58}$ C.-H. Kuo, T. Wyttenbach, C. G. Beggs, and P. R. Kemper, J. Chem. Phys. 92, 4849 (1990)

${ }^{59}$ R. Marx, S. Fenistein, G. Mauclaire, J. Lemaire, and M. Heninger, Int. J. Mass Spectrom. Ion Processes 132, 143 (1994).

${ }^{60}$ E. C. Zipf, Planet. Space Sci. 36, 621 (1988).

${ }^{61}$ Z. Amitay et al., Phys. Rev. A 60, 3769 (1999).

${ }^{62}$ M. Geoghegan, N. G. Adams, and D. Smith, J. Phys. B 24, 2589 (1991).

${ }^{63}$ S. Laubé, L. Lehfaoui, B. R. Rowe, and J. B. A. Mitchell, J. Phys. B 31, 4181 (1998).

${ }^{64}$ J. B. A. Mitchell and H. Hus, J. Phys. B 18, 547 (1985).

${ }^{65}$ T. Mostefaoui et al., J. Phys. B 32, 5247 (1999).

${ }^{66}$ J. R. Peterson et al., J. Chem. Phys. 108, 1978 (1998).

${ }^{67}$ C. H. Sheehan and J. P. St. Maurice, J. Geophys. Res. 109, A03302 (2004).

${ }^{68}$ M. R. Torr and D. G. Torr, Planet. Space Sci. 29, 999 (1981).

${ }^{69}$ J. Semeter et al., J. Geophys. Res. 101, 19683 (1996).

${ }^{70}$ H. Takahashi et al., Planet. Space Sci. 38, 547 (1990).

${ }^{71}$ J. L. Fox, Planet. Space Sci. 34, 1241 (1986). 